Research Article

\title{
Deep Learning-Based Assessment of Adverse Cardiovascular Events in Elderly Patients with Coronary Heart Disease after Percutaneous Coronary Intervention Using Intravascular Ultrasound Images
}

\author{
Shu Wang (D) \\ Cardiovascular Medicine, Daqing Longnan Hospital (The Fifth Affiliated Hospital of Qiqihar Medical University), \\ Daqing 163453, Heilongjiang, China \\ Correspondence should be addressed to Shu Wang; 15813128@stu.wxic.edu.cn
}

Received 14 July 2021; Revised 20 August 2021; Accepted 23 August 2021; Published 11 September 2021

Academic Editor: Gustavo Ramirez

Copyright (c) 2021 Shu Wang. This is an open access article distributed under the Creative Commons Attribution License, which permits unrestricted use, distribution, and reproduction in any medium, provided the original work is properly cited.

\begin{abstract}
This study aimed to analyze the risk factors of adverse cardiovascular events (ACVEs) in elderly patients with coronary heart disease (CHD) after percutaneous coronary intervention (PCI) using the intravascular ultrasound (IVUS) images based on the deep learning of convolutional neural networks (CNNs). This study included 90 patients with coronary heart disease as the research object. All the patients were randomly divided into a control group (group C) and an experimental group (group E), and all were treated with PCI. The patients in group C were diagnosed by angiography, and patients in group E underwent IVUS examination under deep learning. The levels of blood lipids and inflammatory factors between the two groups before and after PCI were compared, and the sensitivity, specificity, and positive predictive value (PPV) were recorded. Compared with angiography diagnosis, ultrasound diagnosis based on deep learning algorithm had higher sensitivity (92.3\% vs. $81.4 \%$ ), specificity (90.1\% vs. 88.6\%), and PPV $(94.8 \%$ vs. $75.3 \%)(P<0.05)$. Compared with group C, patients in group $\mathrm{E}$ had a higher narrowest lesion diameter $(2.54 \pm 0.18 \mathrm{~mm}$ vs. $2.21 \pm 0.19 \mathrm{~mm})$ and detection rate of eccentric plaques $(80.1 \%$ vs. $45.3 \%)(P<0.05)$. High-density lipoprotein cholesterol (HDL-C) after PCI in the two groups was significantly higher than that before surgery, while low-density lipoprotein cholesterol (LDL-C), tumor necrosis factor (TNF), and C-reactive protein (CRP) were significantly lower than those before surgery, and the difference was statistically significant $(P<0.05)$. In short, the ultrasonic detection method based on deep learning algorithm has high sensitivity, specificity, and accuracy for CHD detection; PCI can improve the patient's blood lipid level, relieve the patient's inflammation, and reduce the occurrence of ACVEs in the patient.
\end{abstract}

\section{Introduction}

The expansion of CHD is coronary atherosclerotic heart disease. It is caused by the accumulation of fat in the arterial wall, and the calcium-containing substances in the arterial wall gradually accumulate as the accumulated fat increases and deposits. Local tissues of arterial vascular disease are thickened and hardened, and eventually the lesions form congee-like plaques in the blood vessels with yellow appearance [1]. Calcium-containing substance deposition in the inner wall of blood vessels to form atherosclerosis process, medically known as atherosclerosis [2]. At present, the relevant research on the etiology and pathogenesis of the disease has not yet reached a definitive conclusion. It is generally believed that the occurrence of atherosclerosis is mostly due to the inflammatory reaction in the blood vessel, the damage of vascular endothelial cells, and the mechanism of blood coagulation $[1,3]$. The main incidence of atherosclerosis occurs in adult men over 30 or patients with dyslipidemia, hypertension, diabetes, and severe obesity. It has a high incidence in China, and the incidence increases with age, and the incidence in women is relatively low [4]. The treatment of this disease is mainly through appropriate improvement of the patient's lifestyle and corresponding 
drug treatment (including statins) to achieve the purpose of controlling the development of plaque. In order to prevent the further development of the disease, surgery is required if necessary, including PCI treatment $[5,6]$. If patients with atherosclerotic plaques can be discovered in time and undergo standardized treatment before cerebral infarction occurs, they can generally control the development of the disease, stabilize the plaques, and return to normal life in a short period of time [7].

$\mathrm{CNN}$ is a kind of feedforward neural network, which can effectively reduce the complexity of feedback neural network and can be used to identify some distorted and undeformed two-dimensional graphics such as displacement and scaling [8]. The wide application of deep learning such as CNN in medical image classification can provide convenience for researchers on the basis of traditional research methods $[9,10]$. Image segmentation technology is the main step and operates from processing to analyzing images. Currently, the more commonly used segmentation methods are thresholdbased segmentation methods, region-based segmentation methods, edge-based segmentation methods, and specific theory-based segmentation methods $[11,12]$. In addition, neural network recognition technology has begun to attract attention from all walks of life and is widely used in image segmentation. The neural network has a huge number of connections, and it is easy to introduce spatial information, which can better solve the unevenness and noise in image recognition [13].

In this study, CHD patients were selected as the research objects, diagnosed by angiography and deep learning-based IVUS, and treated with PCI. The two methods were compared in terms of diagnosis accuracy of CHD, incidence of ACVEs after PCI, and high-density lipoprotein cholesterol (HDL-C), LDL-C, tumor necrosis factor (TNF), and CRP before and after PCI, so as to explore the risk factors for ACVEs in elderly CHD patients after PCI using the IVUS images based on deep learning, hoping to provide some data support and theoretical basis for the clinical diagnosis and treatment of coronary heart disease.

\section{Materials and Methods}

2.1. Research Objects and Grouping. $90 \mathrm{CHD}$ patients who were hospitalized from September 2018 to September 2020 were selected as the research objects, aged 40-60 years, including 50 males and 40 females. Patients who voluntarily withdrew and transferred to the hospital were excluded. All patients were randomly divided into a control group (group C) and an experimental group (group E), with 45 cases in each group, and both received PCI treatment. Patients in group $\mathrm{C}$ were diagnosed by angiography; those in group $\mathrm{E}$ were diagnosed by IVUS under deep learning. The study had been approved by the Ethics Committee of the hospital, and the patients and their families had understood the contents and methods of the study and agreed to sign the corresponding informed consent forms.

Inclusion criteria were as follows: patients with clear consciousness and who can cooperate with treatment and sample collection; patients with carotid atherosclerotic plaque confirmed by premenstrual examination; patients with complete clinical data and information; patients with no history of mental illness and emotional stability.

Exclusion criteria were as follows: patients who withdrew and transferred for treatment due to personal reasons; patients with other serious diseases or infectious diseases; patients with hypertension and other diseases or underwent similar surgical treatment; patients suffering from connective tissue diseases or malignant tumors; patients who were unwilling to actively cooperate with treatment.

\subsection{Coronary Artery Ultrasound Examination of Patients.} The two groups of patients underwent carotid artery ultrasound and carotid angiography. They were required to fast one day before the examination and prohibited drinking water for 5 hours before the examination. The patient's heart rate, blood pressure, and other indicators were measured and recorded. The fasting venous blood was collected to detect the blood lipids, and fasting blood glucose (GLU), blood uric acid (BUA), and plasma fibrinogen (FIB) were measured. In addition, the GLU was measured and recorded $2 \mathrm{~h}$ after eating:

Group C: arteriography examination was implemented with an angiography machine to puncture the femoral artery with the Seldinger method. The patient was required to remain in a supine position on the testing table and was disinfected with lidocaine for local anesthesia. First, the common carotid artery on the affected side of carotid atherosclerosis was imaged, and then, the external carotid artery branch was examined with the superselective angiography.

Group E: arterial ultrasound examination was realized with a GE Vivid7 color Doppler ultrasound system with the frequency of $7-10 \mathrm{MHz}$. The tested patient lied supine on the testing table and tilted the head to one side to expose the tested part as much as possible to facilitate inspection. First, the carotid artery was scanned with a lateral probe, which was displayed by two-dimensional ultrasound. The main inspection items included the inner diameter of the blood vessel, whether there was plaque around the blood vessel and the wall of the blood vessel, and determination of the location and size of the plate. After that, longitudinal detection was performed. The patient was required to ensure the anterior and lateral view of the carotid artery for longitudinal detection. The main detection items included detection of the length and thickness of the plaque, continuity of the lumen intima, blood flow and filling degree of the blood vessel, stenosis section, and obstruction section.

2.3. CNN Algorithm. The CNN algorithm mainly referred to a linear operation method of two real-valued functions for algorithmic processing of grid-type data, which were obtained by combining multiple basic simple operation processes. Compared with other neural networks, the CNN had independent connection and calculation methods, but they 
were all based on the backpropagation algorithm, based on which the network parameters were adjusted and optimized:

$$
T(n)=\int_{-\infty}^{+\infty} I(x) O(n-x) Y_{x} .
$$

In CNN, $I(x)$ and $O(x)$ represent the integrable functions on $R$, and the convolution of them is shown in equation (1). In equation (1), $Y(x)$ represents the input and $O(x)$ represents the convolution kernel; then, $T(n)$ represents the feature map as follows:

$$
T(n)=\sum_{x=-\infty}^{+\infty} I(x) O(n-x) .
$$

For computer processing of the discrete data in the image in actual application, both $I(x)$ and $O(x)$ could be defined in discrete variables and their convolutions could be expressed as equation (2). CNN could identify images through sliding window and selective search. The algorithm could first identify whether the window was a target, which could then be identified as the regression of the occurrence probability of each target in image segmentation.

Under normal circumstances, CNN mainly includes convolutional layer, pooling layer, and fully connected layer. The loss function was adopted to optimize the training and update parameters. The convolutional layer was the most critical basic structure in CNN. It mainly implemented the network convolution operation on data: if the input single sample data vector was $x_{i}^{a}=\left[x_{1}, x_{2}, \ldots, x_{m}\right]$, then the output signal $C$ after the convolutional layer calculation was given as follows:

$$
C_{i}^{t, n}=f\left(d_{n}^{t}+\sum_{s=1}^{S} h_{s}^{n} \bullet x_{i+s-1}^{\text {an }}\right) .
$$

In equation (3), $t$ represents the index of the layer, $f$ represents the activation function, which was used to introduce the nonlinear processing to the layer, $d$ represents the deviation term of the $n$th feature map, $S$ represents the size of the convolution kernel, and $h s$ refers to the weight between the $n^{\text {th }}$ feature map and the $s^{\text {th }}$ filter.

The pooling layer was also called the subsampling layer. Pooling could be classified into maximum pooling and average pooling, and the calculation methods of them could be found in the following equations:

$$
\begin{gathered}
P_{i}^{t, n}=\max _{k \in K} C_{i \times L+k}^{t, n}, \\
P_{i}^{t, n}=\frac{1}{|K|} \sum_{k \in K} C_{i \times L+k}^{t, n} .
\end{gathered}
$$

In equations (4) and (5), $C$ represents the input signal, $P$ represents the output signal, $K$ represents the size of the pooling window, and $L$ represents the pooling step size.

The fully connected layer connected each neuron in the output layer and the upper input layer to achieve feature integration. The calculation method can be expressed as follows:

$$
Q_{i}^{t, n}=f\left(d_{n}^{t}+g_{i}^{t, n} \bullet P_{i}^{t, n}\right)
$$

In equation (6), $g$ represents the weight value of the fully connected layer.

In order to solve the unclear lesion edge and insufficient local contrast in the image, the adaptive histogram equalization (HE) method was applied to enhance the image contrast, which could be defined as follows:

$$
f(n)=\frac{255 \times C(n)}{X * X} .
$$

The size of the sliding window is defined as $X * X, f(n)$ is the local mapping function, and $C(n)$ is the cumulative distribution function. Then, the slope of the local mapping function $f(n)$ could be expressed as follows:

$$
\begin{aligned}
K & =\frac{\mathrm{d}(f(n))}{\mathrm{d} i} \\
& =\operatorname{HE}(n) \times \frac{255}{X * X} .
\end{aligned}
$$

When the maximum local mapping function slope was $K_{\max }$, the maximum histogram height was $L_{\max }$, which can be calculated as follows:

$$
\begin{aligned}
L_{\max } & =K_{\max } \times \frac{X * X}{255}, \\
O_{n} & =T\left(\sum_{m=1}^{t} Q_{n m} I_{n}-\gamma_{n}\right) .
\end{aligned}
$$

The calculation model of the neural network-MP mode was as in equation (10). $O_{n}$ is the output of the neuron, $I_{n}(n$ stands for $1,2,3, \ldots, n)$ is the input signal, $T$ refers to the neuron activation function, $Q_{n m}$ refers to the connection strength between two neurons, and $\gamma_{n}$ represents the threshold of activating neurons:

$$
\operatorname{Wei}(T, F)=\sum_{n=1}^{m} W_{n} f\left(T_{n}, F_{n}\right) .
$$

All levels of weight values were assigned through the weighted loss function, and the weight of loss could be calculated with equation (11). Here, $n$ is the selected sample size, $T$ is the true value input, $F$ is the predicted value input, and $f$ refers to the loss function.

2.4. Observation Index. The basic information of the two groups of patients was compared, including average age, systolic blood pressure, diastolic blood pressure, and serum thyroglobulin (TG) content; the sensitivity, specificity, and PPV of the two diagnostic methods were also compared for the two groups of patients; adverse events after PCI were recorded. The incidence of the disease, diameter of the narrowest part of the lesion detected by the two methods, the detection rate of eccentric plaques, and the detection rate of concentric plaques were calculated; the blood lipids (HDL-C and LDL-C) of the two groups before and after PCI and levels of inflammatory factors (TNF and CRP) were also compared. 
2.5. Statistical Methods. The data processing of this study was analyzed by SPSS 23.0 version statistical software, the measurement data were indicated as the mean \pm standard deviation $(x \pm s)$, and the count data were displayed as the percentage. Pairwise comparison was implemented with the analysis of variance. $P<0.05$ indicated that the difference was statistically meaningful.

\section{Results}

3.1. Angiography and Ultrasound Images of the Patient with CHD. Figure 1(a) shows coronary angiography of patients with coronary heart disease. The left and right coronary arteries were normal in shape and distribution. The stenosis of the proximal anterior descending branch and the proximal diagonal branch was about $95 \%$ and $70 \%$, respectively. Figure 1(b) shows an image of a CHD patient diagnosed by ultrasound. It showed that the inner medium of the coronary artery wall was thicker than $1.2 \mathrm{~mm}$, which was linear and uneven with enhanced echo; there were eccentrically distributed hypoechoic lipid plaques in the vessel wall, and the plate area was large; the area of the blood vessel in the plate distribution was larger; the stenosis rate of the official cavity area was obviously increased, showing obvious positive remodeling, and superficial calcification and punctate calcification could be found.

3.2. Comparison on Basic Information of the Two Groups of Patients. The two groups of CHD patients were diagnosed by ultrasound and angiography detection methods, and related data and basic information of the patients were collected and recorded. Figure 2 shows the average age and systolic blood pressure of the two groups of patients. It is illustrated that there was no visible difference in age between group $\mathrm{C}$ and group $\mathrm{E}$ patients $(45 \sim 55$ years old) $(P>0.05)$; the systolic blood pressure of group $\mathrm{C}(150.2 \pm 12.38 \mathrm{mmHg})$ and group $\mathrm{E}$ patients $(149.38 \pm 14.6 \mathrm{mmHg})$, respectively, was not different significantly $(P>0.05)$.

The diastolic blood pressure and serum thyroglobulin (TG) content of the two groups were tested and compared. The results in Figure 3 illustrated that the diastolic blood pressure of group C and group $\mathrm{E}$ was $79.38 \pm 8.2 \mathrm{mmHg}$ and $80.1 \pm 7.8 \mathrm{mmHg}$, respectively, and the TG was $1.41 \pm 0.38 \mathrm{mmol} / \mathrm{L}$ and $1.42 \pm 0.39 \mathrm{mmol} / \mathrm{L}$, respectively. Hence, there was no obvious difference in diastolic blood pressure and serum TG content between two groups $(P>0.05)$.

3.3. Sensitivity, Specificity, and PPV of the Two Diagnostic Methods for the Two Groups of Patients. As shown in Figure 4, the angiography and deep learning algorithm-based ultrasonic diagnostic methods were compared in terms of sensitivity, specificity, and PPV of CHD diagnosis. The results revealed that the sensitivity, specificity, and PPV of group C were $81.4 \%, 88.6 \%$, and $75.3 \%$, respectively, while those in group E was $92.3 \%, 90.1 \%$, and $94.8 \%$, respectively. Thus, those in group E were higher sufficiently in contrast to group $\mathrm{C}(P<0.05)$.
3.4. Incidence of Adverse Events after PCI. Figure 5 illustrates the incidence of ACVEs after PCI. It revealed that some patients suffered from heart failure (5 cases), myocardial infarction (3 cases), typical angina (10 cases), and sudden cardiac death (2 cases). Among them, the typical angina accounted for the highest proportion, followed by heart failure and myocardial infarction.

3.5. Comparison on Detection Results of CHD in the Two Methods. Figure 6 discloses the comparison of the diameter of the narrowest part of the lesion detected by two methods. It was obvious that diameters of the narrowest lesions detected by group C and group E were $2.21 \pm 0.19 \mathrm{~mm}$ and $2.54 \pm 0.18 \mathrm{~mm}$, respectively, with a significant higher value in group $\mathrm{E}(P<0.05)$.

As illustrated in Figure 7, the detection rates of eccentric plaque by IVUS and coronary angiography were compared. The comparison results proved that the detection rates of eccentric plaques in group C and group E were $45.3 \%$ and $80.1 \%$, respectively, with a dramatic difference $(P<0.05)$.

Figure 8 shows the comparison results of the detection rate of centripetal plaque by IVUS and coronary angiography. It indicated that the detection rates in group $\mathrm{C}$ $(58.2 \%)$ were obviously lower than those in group E $(40.1 \%)$ $(P<0.05)$.

3.6. Levels of Blood Lipids and Inflammatory Factors in the Two Groups before and after PCI. The HDL-C levels between the two groups of patients before and after PCI were compared, as given in Figure 9. The preoperative and postoperative $\mathrm{HDL}-\mathrm{C}$ levels in group $\mathrm{C}$ were $0.98 \pm 0.06 \mathrm{mmol} / \mathrm{L}$ and $1.44 \pm 0.05 \mathrm{mmol} / \mathrm{L}$, respectively; those in group $\mathrm{E}$ were $0.99 \pm 0.05 \mathrm{mmol} / \mathrm{L}$ and $1.32 \pm 0.03 \mathrm{mmol} / \mathrm{L}$, respectively. Thus, the preoperative HDL-C levels of the two groups were lower observably than those after PCI $(P<0.05)$.

The LDL-C levels between the two groups of patients before and after PCI were compared, as illustrated in Figure 10. The LDL-C levels before and after PCI were $4.56 \pm 0.13 \mathrm{mmol} / \mathrm{L}$ and $2.87 \pm 0.24 \mathrm{mmol} / \mathrm{L}$ in group $\mathrm{C}$, respectively; those were $4.58 \pm 0.21 \mathrm{mmol} / \mathrm{L}$ and $2.81 \pm 0.22 \mathrm{mmol} / \mathrm{L}$ in group $\mathrm{E}$, respectively. The preoperative LDL-C levels before PCI in the two groups were significantly higher than those after PCI $(P<0.05)$.

The TNF levels in patients of two groups before and after PCI were compared. As revealed in Figure 11, the TNF levels before and after the PCI were $135.87 \pm 5.84 \mathrm{ng} / \mathrm{L}$ and $79.44 \pm 4.33 \mathrm{ng} / \mathrm{L}$ in group $\mathrm{C}$, respectively; those in group $\mathrm{E}$ were $134.95 \pm 6.21 \mathrm{ng} / \mathrm{L}$ and $78.73 \pm 4.98 \mathrm{ng} / \mathrm{L}$, respectively. It was clear that the TNF levels in both groups decreased significantly after the PCI $(P<0.05)$.

As shown in Figure 12, the CRP level in groups $\mathrm{C}$ and $\mathrm{E}$ before PCI was $78.3 \pm 3.87 \mathrm{mmol} / \mathrm{L}$ and $77.9 \pm 3.21 \mathrm{mmol} / \mathrm{L}$, respectively; those in groups $\mathrm{C}$ and $\mathrm{E}$ after the PCI was $56.9 \pm 3.54 \mathrm{mmol} / \mathrm{L}$ and $57.2 \pm 3.76 \mathrm{mmol} / \mathrm{L}$, respectively. Thus, it disclosed that the CRP levels in both groups of patients before the PCI were higher significantly in contrast to the levels after PCI $(P<0.05)$. 


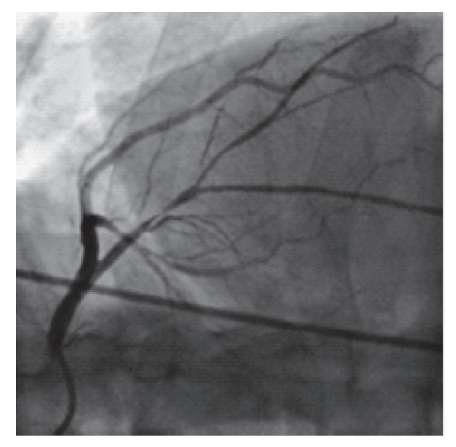

(a)

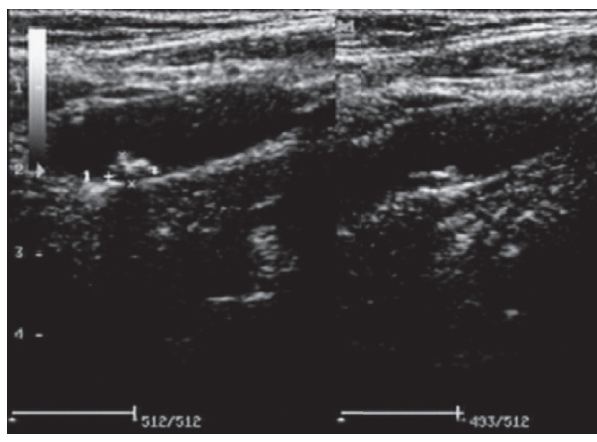

(b)

FIgURE 1: Angiography and ultrasound images of the patient with CHD. (a) Coronary angiography. (b) Coronary artery ultrasound images.

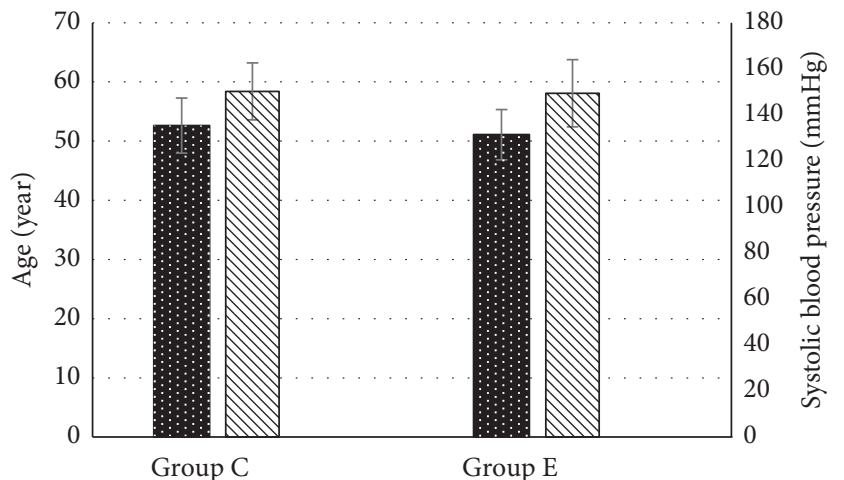

Age

$\$$ Systolic blood pressure

FIgURE 2: Average age and systolic blood pressure of the two groups of patients.

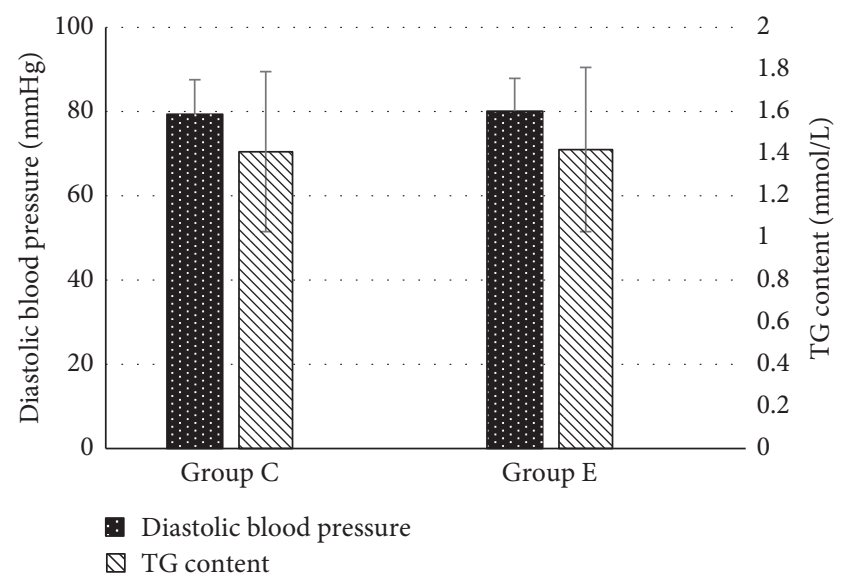

Figure 3: Comparison on diastolic blood pressure and TG contents.

\section{Discussion}

CHD should be treated with drugs or surgery according to the degree of atherosclerotic plaque stenosis in patients [14]. Arteries mainly provide blood supply to the front of the brain [15]. If there is any stenosis or hyperplasia, it will cause

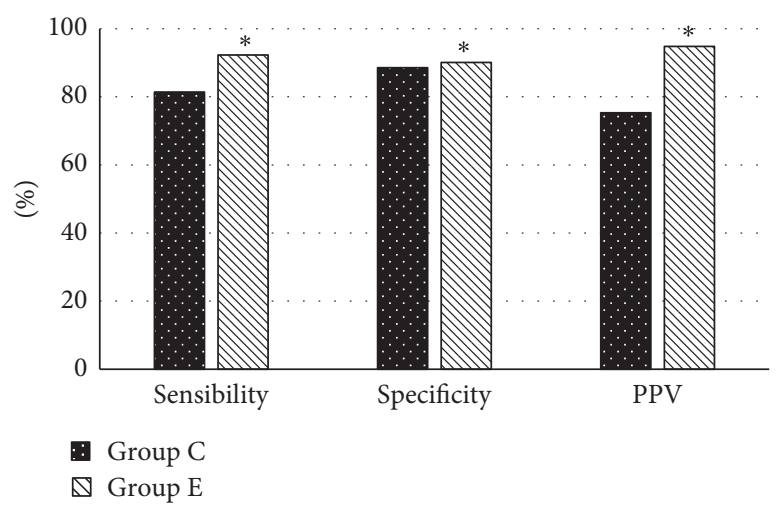

FIGURe 4: Comparison on sensitivity, specificity, and PPV in CHD diagnosis of two methods. ${ }^{*}$ The difference was visible in contrast to group $\mathrm{C}(P<0.05)$.

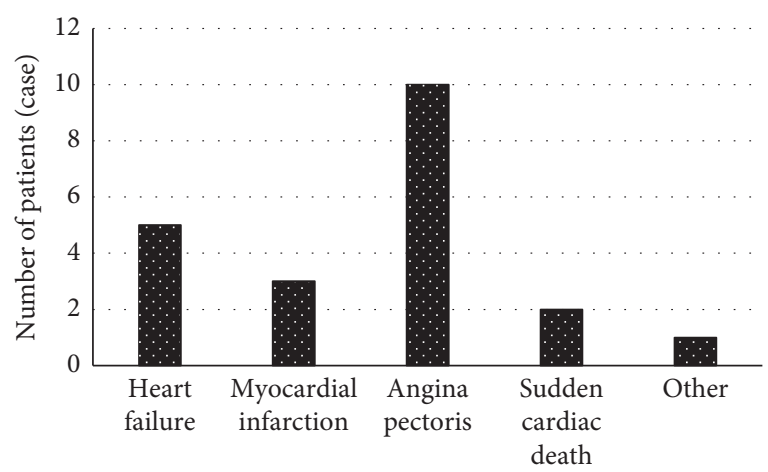

FIGURE 5: Incidence of ACVEs after PCI.

problems with the blood supply to the brain. If it lasts for a long time, a stroke may occur, such as speech disorders, mobility problems, and swallowing disorders [16]. If patients want to treat coronary heart disease, they must first determine the degree of stenosis caused by carotid atherosclerosis. If the atherosclerotic plaque is greater than $50 \%$ or greater than $70 \%$, a stent or carotid artery is required [17].

This study included 90 patients with coronary heart disease as the research object. All the patients were randomly divided into a control group (group C) and an experimental group (group E), and all were treated with PCI. The patients 


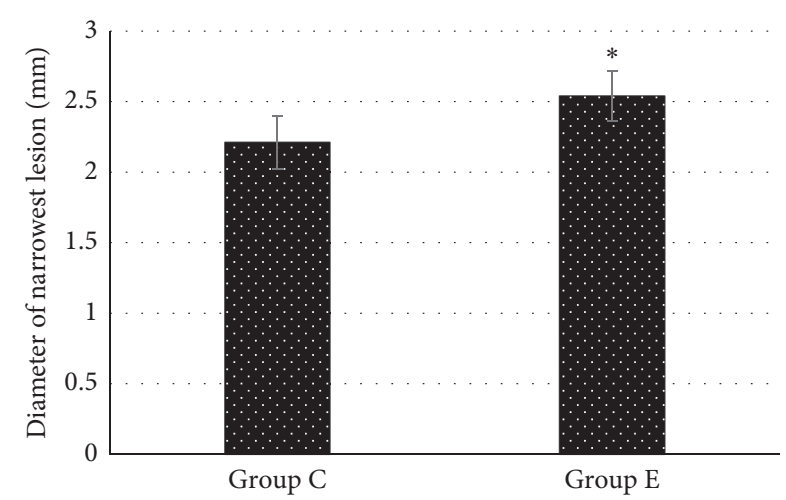

FIGURE 6: Comparison of the diameter of the narrowest part of the lesion detected by two methods. ${ }^{*}$ The difference was visible in contrast to group $\mathrm{C}(P<0.05)$.

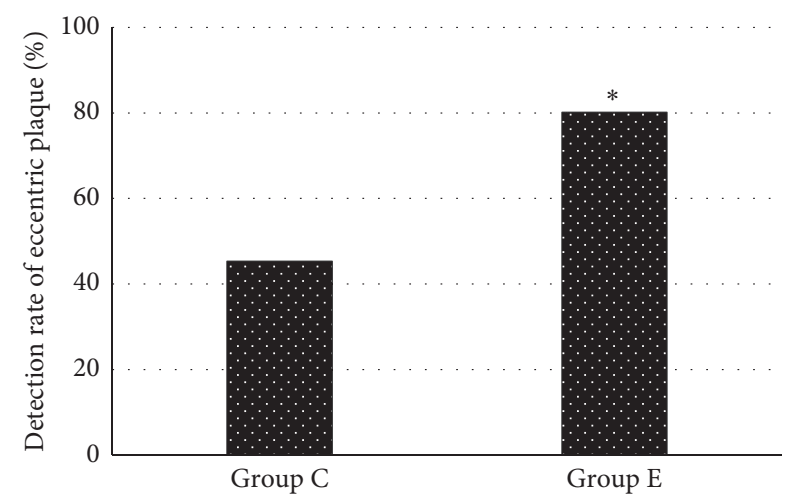

FIGURE 7: Detection rates of eccentric plaque by IVUS and coronary angiography. ${ }^{*}$ The difference was visible in contrast to group C $(P<0.05)$.

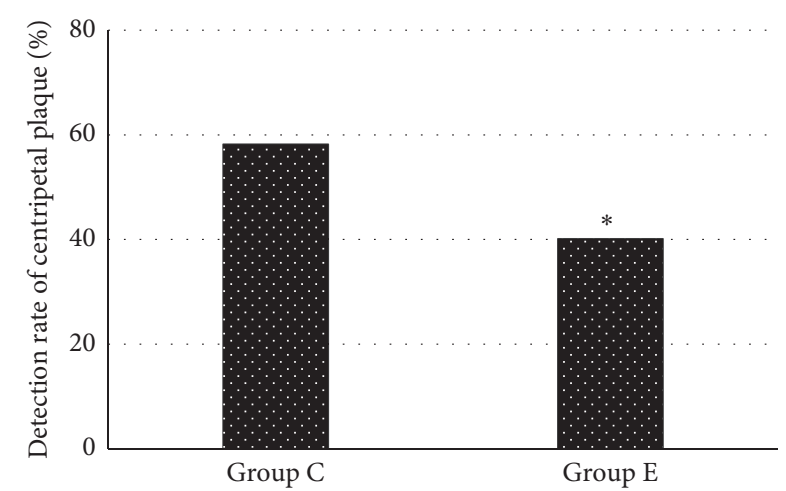

FIGURE 8: Detection rates of centripetal plaque by IVUS and coronary angiography. ${ }^{*}$ The difference was visible in contrast to group C $(P<0.05)$.

in group $\mathrm{C}$ were diagnosed by angiography; patients in group E underwent IVUS examination under deep learning. The basic information of the two groups of patients was compared. The incidence of adverse events after PCI was recorded. The levels of blood lipids and inflammatory factors in the two groups before and after PCI and sensitivity, specificity, and PPV of the two diagnostic methods in the two groups of patients were compared. The diameter of the

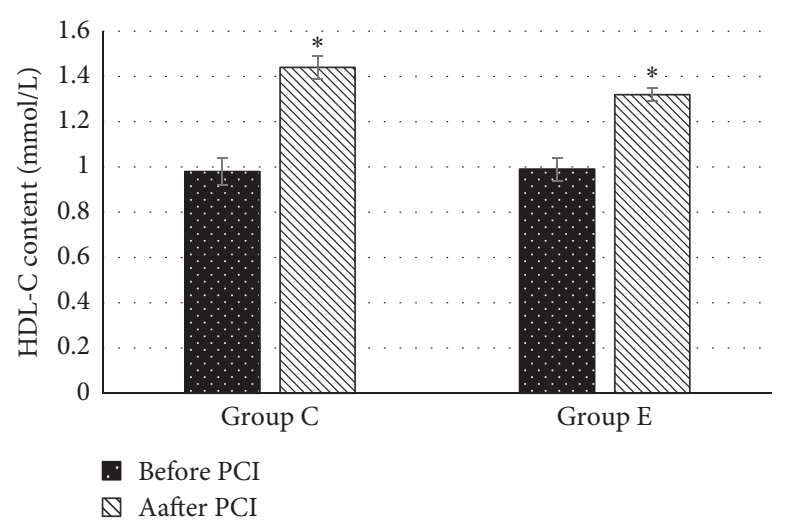

Figure 9: Comparison on HDL-C content in patients before and after PCI. *The statistical difference was visible in contrast to the value before PCI $(P<0.05)$.

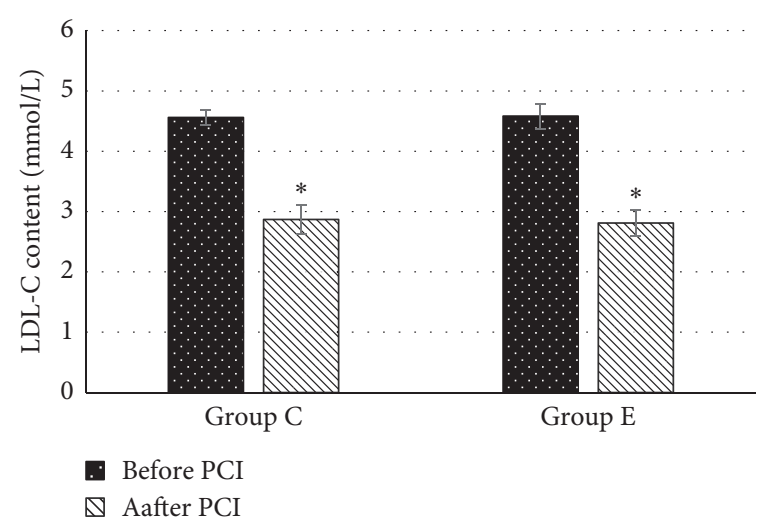

Figure 10: Comparison on LDL-C content in patients before and after PCI. ${ }^{*}$ The statistical difference was visible in contrast to the value before PCI $(P<0.05)$.

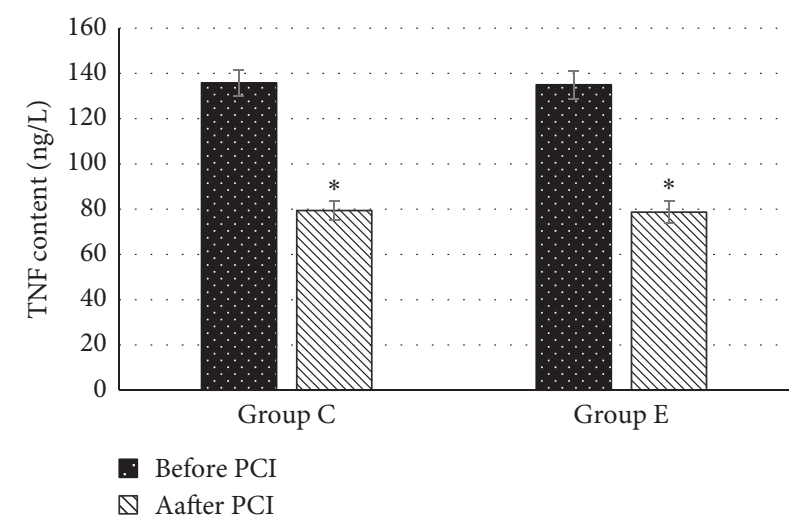

FIgURE 11: Comparison on TNF level in patients before and after PCI. ${ }^{*}$ The statistical difference was visible in contrast to the value before PCI $(P<0.05)$.

narrowest part of the detected lesions and the detection rate of eccentric plaques and centripetal plaques were calculated. Angina pectoris (10 cases) was the most common after PCI, followed by heart failure ( 5 cases) and myocardial infarction ( 3 cases). The sensitivity (92.3\%), specificity (90.1\%), and accuracy $(94.8 \%)$ of ultrasound diagnosis based on deep 


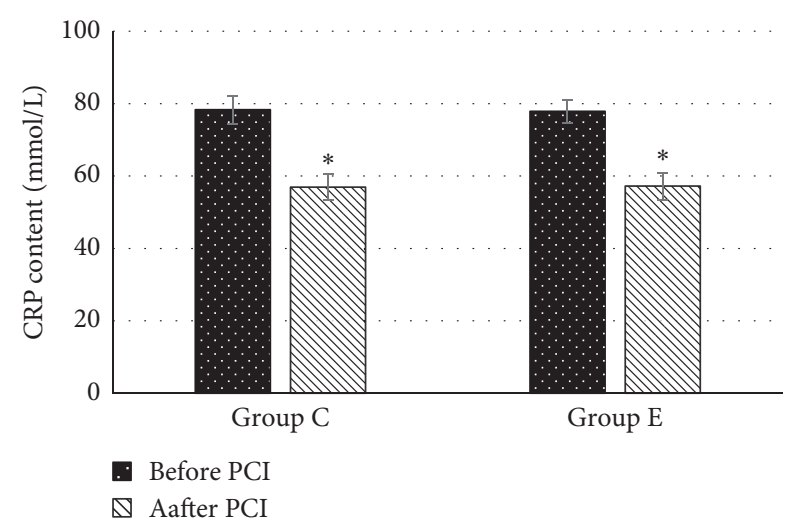

Figure 12: Comparison on CRP level in patients before and after PCI. *The statistical difference was visible in contrast to the value before PCI $(P<0.05)$.

learning algorithms were significantly higher than those of angiographic diagnosis $(81.4 \%, 88.6 \%$, and $75.3 \%)$, and the difference was statistically significant $(P<0.05)$.

The narrowest lesion diameter $(2.54 \pm 0.18 \mathrm{~mm})$ and the detection rate of eccentric plaques $(80.1 \%)$ in group $\mathrm{E}$ were significantly higher than those in group C $(2.21 \pm 0.19 \mathrm{~mm}$ and $45.3 \%)$, while the detection rate of concentric plaques (40.1\%) was significantly lower than that of group C (58.2\%), and the difference was statistically significant $(P<0.05)$. This was consistent with the results of Zhu et al. [18]. The application of convolutional neural networks to the imaging diagnosis of cardiovascular and cerebrovascular diseases has significantly improved the sensitivity and accuracy of diagnosis compared with traditional diagnostic methods.

HDL-C $(1.44 \pm 0.05 \mathrm{mmol} / \mathrm{L}$ and $1.32 \pm 0.03 \mathrm{mmol} / \mathrm{L})$ after PCI in the two groups was significantly higher than that before surgery $(0.98 \pm 0.06 \mathrm{mmol} / \mathrm{L}$ and $0.99 \pm 0.05 \mathrm{mmol} / \mathrm{L})$, while postoperative LDL-C $(2.87 \pm 0.24 \mathrm{mmol} / \mathrm{L}$ and $2.81 \pm 0.22 \mathrm{mmol} / \mathrm{L})$ was significantly lower than that before surgery $(4.56 \pm 0.13 \mathrm{mmol} / \mathrm{L}$ and $4.58 \pm 0.21 \mathrm{mmol} / \mathrm{L})$, and the difference was statistically significant $(P<0.05)$. TNF $(79.44 \pm 4.33 \mathrm{ng} / \mathrm{L}$ and $78.73 \pm 4.98 \mathrm{ng} / \mathrm{L})$ and CRP $(56.9 \pm 3.54 \mathrm{mmol} / \mathrm{L}$ and $57.2 \pm 3.76 \mathrm{mmol} / \mathrm{L})$ after PCI in the two groups were significantly lower than preoperative TNF $(135.87 \pm 5.84 \mathrm{ng} / \mathrm{L}$ and $134.95 \pm 6.21 \mathrm{ng} / \mathrm{L})$ and CRP $(78.3 \pm 3.87 \mathrm{mmol} / \mathrm{L}$ and $77.9 \pm 3.21 \mathrm{mmol} / \mathrm{L})$, and the difference was statistically significant $(P<0.05)$. This was consistent with the findings of Goertz [19]. PCI can improve the patient's blood lipid level and alleviate inflammation in patients with coronary heart disease after treatment, which may reduce the incidence of ACVEs in patients.

\section{Conclusion}

This study included patients with CHD as the research object. All patients were randomly divided into a control group (group C) and an experimental group (group E). All patients were treated with PCI. The patients in group $\mathrm{C}$ were diagnosed by angiography, and patients in group $\mathrm{E}$ underwent IVUS examination under deep learning. The accuracy, incidence of ACVEs after PCI, and levels of HDL-C,
LDL-C, TNF, and CRP before and after PCI for patients in two groups were compared. The results suggested that the ultrasound detection method based on deep learning algorithm showed high sensitivity, specificity, and accuracy in detecting CHD; PCI can improve the patient's blood lipid level and relieve the patient's inflammation, which may reduce the occurrence of ACVEs in the patient. However, the sample size selected was small, which might have an impact on the experimental results, so that the representativeness was low. Therefore, the sample size will be increased in subsequent experiments, so as to further analyze and discuss the risk factors of ACVEs for CHD patients after PCI using the IVUS image based on the deep learning. In short, this study provided some data support and theoretical basis for clinical diagnosis and treatment of CHD.

\section{Data Availability}

The data used to support the findings of this study are available from the corresponding author upon request.

\section{Conflicts of Interest}

The author declares that there are no conflicts of interest.

\section{References}

[1] A. S. Pushkin, "Personified monitoring of acute coronary syndrome and its outcomes in elderly patients. Report 2 . Invasive diagnostic and treatment methods (review)," Advances in Gerontology, vol. 32, no. 6, pp. 976-984, 2019.

[2] Q.-h. Shang, H. Xu, X.-y. Lu, C. Wen, D.-z. Shi, and K.-j. Chen, "A multi-center randomized double-blind placebo-controlled trial of Xiongshao Capsule (芎节胶囊) in preventing restenosis after percutaneous coronary intervention: a subgroup analysis of senile patients," Chinese Journal of Integrative Medicine, vol. 17, no. 9, pp. 669-674, 2011.

[3] A. Boening, L. Assling-Simon, M. Heep, K. Boengler, B. Niemann, and P. Grieshaber, "Buckberg's blood cardioplegia for protection of adult and senile myocardium in a rat in vitro model of acute myocardial infarction," Experimental Gerontology, vol. 104, pp. 98-104, 2018.

[4] A. N. Shishkevich, V. N. Kravchuk, K. L. Kozlov, I. B. Oleksiuk, S. S. Mikhaŭlov, and G. G. Khubulava, "Endovascular treatment of bifurcation lesions of the coronary arteries in patients of elderly age: a review of the literature No 1," Advances in Gerontology, vol. 27, no. 3, pp. 510-518, 2014.

[5] E. Leen, M. Averkiou, M. Arditi et al., "Dynamic contrast enhanced ultrasound assessment of the vascular effects of novel therapeutics in early stage trials," European Radiology, vol. 22, no. 7, pp. 1442-1450, 2012.

[6] Y. J. Sun, J. P. Qiu, P. X. Zhu et al., "Long-term outcomes of multiple arterial off-pump coronary artery bypass grafting," Zhonghua Yixue Zazhi, vol. 99, no. 14, pp. 1053-1057, 2019.

[7] G. G. Khubulava, K. L. Kozlov, S. S. Mikhaŭlov, A. N. Shishkevich, and I. B. Oleksiuk, "Features of antiagregant and anticoagulant therapy at a hybrid revascularization of a myocardium in elderly and senile patients," Advances in Gerontology, vol. 26, no. 4, pp. 685-688, 2013.

[8] Z. Lv and W. Xiu, "Interaction of edge-cloud computing based on SDN and NFV for next generation IoT," IEEE Internet of Things Journal, vol. 7, no. 7, pp. 5706-5712, 2019. 
[9] C. T. P. Lewis, R. L. Stephens, J. L. Cline, and C. M. Tyndal, "Concurrent minimally invasive aortic valve replacement and coronary artery bypass via limited right anterior thoracotomy," Innovations: Technology and Techniques in Cardiothoracic and Vascular Surgery, vol. 10, no. 4, pp. 273-275, 2015.

[10] A. Xie, T. Belcik, Y. Qi et al., "Ultrasound-mediated vascular gene transfection by cavitation of endothelial-targeted cationic microbubbles," Journal of the American College of Cardiology: Cardiovascular Imaging, vol. 5, no. 12, pp. 12531262, 2012.

[11] R. Feng, M. Badgeley, J. Mocco, and E. K. Oermann, “Deep learning guided stroke management: a review of clinical applications," Journal of Neurointerventional Surgery, vol. 10, no. 4, pp. 358-362, 2018.

[12] A. Hosny, C. Parmar, T. P. Coroller et al., "Deep learning for lung cancer prognostication: a retrospective multi-cohort radiomics study," PLoS Medicine, vol. 15, no. 11, Article ID e1002711, 2018.

[13] F. Liu, H. Jang, R. Kijowski, T. Bradshaw, and A. B. McMillan, "Deep learning MR imaging-based attenuation correction for PET/MR imaging," Radiology, vol. 286, no. 2, pp. 676-684, 2018.

[14] R. J. G. Van Sloun, L. Demi, S. G. Schalk et al., "Contrastenhanced ultrasound tractography for $3 \mathrm{D}$ vascular imaging of the prostate," Scientific Reports, vol. 8, no. 1, Article ID 14640, 2018.

[15] E. Abma, E. Stock, W. De Spiegelaere et al., "Power Doppler ultrasound and contrast-enhanced ultrasound demonstrate non-invasive tumour vascular response to anti-vascular therapy in canine cancer patients," Scientific Reports, vol. 9, no. 1 , p. 9262, 2019.

[16] J. Rebling, H. Estrada, S. Gottschalk et al., "Dual-wavelength hybrid optoacoustic-ultrasound biomicroscopy for functional imaging of large-scale cerebral vascular networks," Journal of Biophotonics, vol. 11, no. 9, Article ID e201800057, 2018.

[17] Y. Wang, Z. Xuan, X. Zheng et al., "Efficacy of endostar combined with transcatheter arterial chemoembolization and analysis of vascular endothelial factor and C-reactive protein levels in patients with advanced hepatocellular carcinoma under contrast enhanced ultrasound," Journal of B.U.ON. : Official Journal of the Balkan Union of Oncology, vol. 24, no. 6, pp. 2394-2401, 2019.

[18] Y.-C. Zhu, Y. Zhang, S.-H. Deng, and Q. Jiang, "A prospective study to compare superb microvascular imaging with grayscale ultrasound and color Doppler flow imaging of vascular distribution and morphology in thyroid nodules," Medical Science Monitor, vol. 24, pp. 9223-9231, 2018.

[19] D. E. Goertz, "An overview of the influence of therapeutic ultrasound exposures on the vasculature: high intensity ultrasound and microbubble-mediated bioeffects," International Journal of Hyperthermia, vol. 31, no. 2, pp. 134-144, 2015. 